\title{
EL TERRITORIO Y LA CIUDAD Y LA ENCRUCIJADA EN EL SIGLO XXI
}

\author{
Dr. Daniel González Romero. \\ Director de la "División de Artes y Humanidades" y \\ Director del Programa de Doctorado "Ciudad, Territorio y Sustentabilidad". \\ Universidad de Guadalajara (México).
}

Remisión Artículo: 22-5-2007

Palabras Clave: Territorio, la ciudad, América Latina, mundo real.

Resumen: El tema que me propongo abordar es quizá el que más nos ha ocupado, apasionado, y en el que la ciudad y el territorio como un lugar de confirmación de contextos, de ideologías, de imaginarios y utopías. En esta intervención, América Latina se convierte en el eje central en el que se encadenan ciudades y territorios. Si bien no trato aquí detalles o particularidades en razón del tiempo y la extensión. Es decir, se trata de realizar un breve examen de nuestra región, verla en lo profundo y amplio de su realidad a través de una mirada crítica, en un momento en el que como pocas veces hay una discusión apasionada, intensa, porque según parece desde el repaso de muchos analistas, hemos perdido el rumbo respecto de lo que nuestra región es. Eso hace necesario detenernos en este ejercicio ya que la región encuentra hoy entretejido su futuro, mucho más que en otros momentos, a los sucesos que reestructuran los contextos del devenir de la humanidad. Entonces entre las preguntas fundamentales resalta la necesidad de distinguir, de cuestionar con énfasis: ¿Que es hoy América Latina y hacia donde va?

Introducción: Debo agradecer la invitación a los organizadores de este congreso y a quienes lo han propiciado desde su origen para estar aquí entre ustedes. A la empresa LABEIN y al compañero y amigo Josep Roca, lo mismo que a Roberto Lira y a las Instituciones que han propiciado esta interesante iniciativa, la que ha venido a ocupar un lugar en el mundo de las ideas y del debate sobre los temas que en este se tratan. Entiendo en esta dimensión la importancia que lo virtual viene tomando en el mundo real. Recuerdo la frase aquella de que lo que no esta en la red es virtual. Sin embargo lo virtual en mi intervención acude a la imaginación y al conciencia de todos los que una manera u otra comprometemos nuestra actuación en el contexto de los social, de la realidad concreta que al fin y al cabo lo virtual también tiene algo de eso a pesar de que apunta a la exactitud del conocimiento, para acometer con objetividad la realidad que experimenta la humanidad en su versión territorial, en su noción de región, y en la consideración vital del planeta.

Por otra parte el intercambio y la cooperación que desde hace un buen número de años hemos mantenido un buen numero de sus Universidades, así como otros organismos y personas de España, con Europa en general, que incluyen participación en el programa ALFA y en la Comisión Europea en el Programa ALBAN, hacen que aprecie en todo lo que vale esta generosa invitación. Es por ello que, con una disculpa al margen por no ser un especialista en el área de las tecnologías informáticas, puedo decir que no podía dejar de darme el gusto de aceptar esta invitación, propiciada por el amigo Josep Roca, con quien compartí el segundo congreso en Concepción, Chile, y más recientemente un Coloquio-Taller en Guadalajara, para expresar a ustedes una relación de ideas que acometen lo virtual como una especie de irrealidad alejada de las tecnologías y alojada entre las brumas de la realidad, que parece desapercibida entre las condiciones que impone el proceso de globalización en construcción, que marca entre sorprendentes avances de la ciencia y la tecnología, de la denominada 
sociedad del conocimiento, los rumbos de un cierto-incierto futuro para grandes sectores de la sociedad.

El tema que me propongo abordar es quizá el que más nos ha ocupado, apasionado, y en el que la ciudad y el territorio tienen un lugar de confirmación de contextos, de ideologías, de imaginarios y utopías. Y esto no es solo por una cuestión de interés académico o curiosidad, sino porque ha sido y es un compromiso no solo de forma. Es por eso que en esta intervención, América Latina se convierte en el eje central en el que se encadenan ciudades y territorios. Si bien no trato aquí detalles o particularidades en razón del tiempo y la extensión. Es decir, se trata de realizar un breve examen de nuestra región, verla en lo profundo y amplio de su realidad a través de una mirada crítica, en un momento en el que como pocas veces hay una discusión apasionada, intensa, porque según parece desde el repaso de muchos analistas, hemos perdido el rumbo respecto de lo que nuestra región es. Eso hace necesario detenernos en este ejercicio ya que la región encuentra hoy entretejido su futuro, mucho más que en otros momentos, a los sucesos que reestructuran los contextos del devenir de la humanidad. Entonces entre las preguntas fundamentales resalta la necesidad de distinguir, de cuestionar con énfasis: ¿Que es hoy América Latina y hacia donde va?

Este tema cobra singular significación conforme los sucesos se agolpan en la memoria, es por eso que este foro, como otros en los que he participado últimamente, nos permite llevar más allá de nuestras fronteras, a los lugares y momentos como este en el que participan destacados especialistas, las interrogaciones que se mezclan y articulan en la comprensión de un territorio que se inscribe entre los de merecen atención, no solo por su historia, por su potencial, por su unidad cultural, si no porque es un punto neurálgico del devenir de millones de seres humanos y de su vinculo con la salud social de muchos más, aún de aquellos que deambulan entre el relato de la realidad o si se quiere las realidades del continente latinoamericano, virtuales desde la mirada del que esquiva la desigualdad, realidades que no son ajenas a las que envuelven hoy día al conjunto de las culturas y las economías de los países del mundo, alcanzadas en mayor o menor grado por la construcción virtual e impulso de realidades operables para la cimentación de escenarios futuros.

Para compartir estas reflexiones con ustedes me permito seguir las ideas y trabajos de Luís Maira tomar sus palabras y partir así, entonces, como lo explica, haciendo "un corte en el tiempo, para ver de que manera hemos estimado en los últimos 50 años este largo proceso y ver en éste examen comparativo cuán parecida o distinta es nuestra América Latina en este medio siglo", dicho de otra forma, ver en perspectiva nuestra región continental, asumiendo el compromiso de la versión posible que resulte.

"A principios de los sesentas América Latina era para nosotros los académicos, investigadores y para la comunidad en general, y hasta para los partidos políticos una región muy homogénea. Mirábamos los 20 países latinoamericanos, como países relativamente semejantes. Ninguno se había despegado demasiado de los demás y en promedio eran, de cuerdo a los marcos conceptuales de la época, un grupo de países subdesarrollados que aspiraban a situarse en procesos de "vías de desarrollo" y alcanzar de ésta manera condiciones de ahorro, inversión, innovación tecnológica", de adelantos de lo que significaba en aquellos tiempo el termino "progreso", en el marco de las ideas dominantes de aquellos años sobre que era el desarrollo, que nos permitiera dar el salto cualitativo para llegar a condiciones atributivas y mejorar la vida de todos sus habitantes.

Era una gran extensión territorial marcada por la esperanza y por el deseo de un porvenir lleno de mejores horizontes para el conjunto de nuestros países. 
A principios de los años sesenta, por marcar una fecha, había en América Latina la idea de un territorio homogéneo, con un sentido de identidad y un sentimiento bastante optimista sobre el futuro. Pero lo curiosos era que eran optimistas, las derechas y las izquierdas aunque cada uno por razones distintas. Así en los años siguientes al estallido de la Revolución Cubana, prevalecía la idea muy difundida en un trabajo de gran impacto en ese tiempo "Las etapas del desarrollo económico", de Walt Whitman Rostow, que consideraba que, "Es posible clasificar todas las sociedades, teniendo en cuenta sus aspectos económicos, en cinco categorías: sociedad tradicional, precondiciones para el despegue hacia un crecimiento autosostenido, camino hacia la madurez y etapa de alto consumo.... Estas etapas no son solo descriptivas; no son una mera forma de generalizar las observaciones de ciertos hechos sobre la secuencia del desarrollo en sociedades modernas, sino que tienen su propia lógica interna y continuidad. Estas etapas constituyen finalmente tanto una teoría sobre el crecimiento económico como una teoría más general (aunque todavía muy parcial) de toda la historia moderna. (W.W Rostow, The Stages of Economic Growth, A Non-Communist Manifiesto (Londres: Cambridge University Press, 1960).

De esta manera, con todo y los temores y reacciones ligadas a los planes de control de los Estados Unidos, se había establecido una especie de analogía conceptual que alimentaba el pensamiento de la derecha latinoamericana con la tesis de que existían posibilidades de progreso para el continente, en una suerte de "panamericanismo" que invadía los tiempos, nutrida en tesis de las etapas.

Las derechas consideraban que había una especie de fórmula, de una cierta receta para que los países subdesarrollados, pobres y atrasados, repitieran el camino de los que eran ya países desarrollados: Estados Unidos, Europa, Australia, Nueva Zelanda, y que por lo tanto se trataba de llevar a cabo en la lógica del proceso, de manera inteligente, con el apoyo de los más fuertes, pero con una iniciativa interna firme, una serie de políticas públicas y de estrategias para que con toda seguridad siguiendo el ejemplo de lo que los otros habían realizado, nuestros países pudiesen pasar en un plazo de quince a veinte años de ser pobres a más o menos prósperos, dejar de ser subdesarrollados y pasar con decoro al grupo de una respetable economía y poder, en esos pasos, integrarse en lo posible al proceso de los de mejor fortuna, aunque tal cosa fuese en el vagón de cola del tren del primer mundo. (Eran aquellos tiempos de la NASA, del primer viaje a la luna, de la construcción de Brasilia, la aceleración del crecimiento demográfico, la vía de la industrialización, y también los Chile y Allende, de Cuba, y así de un cierto respiro oxigenado para la democracia). "Las izquierdas por otras razones eran también optimistas, especialmente luego del triunfo de la Revolución Cubana, en 1959, experiencia que se convirtió en un parte aguas en la historia de la América Latina. Fue en su momento este período uno de enorme esperanza, de pensar en la gran revolución latinoamericana para casi prácticamente todos los sectores progresistas de las naciones".

El triunfo de Fidel, El Che, Camilo, y la creciente relación de movimientos en centro y Sudamérica, consolidaba la idea de que era posible construir una sociedad distinta y aspirar a mejores condiciones para todos, desde un lugar, desde una aspiración, que partía del triunfo logrado a noventa millas de los Estados Unidos, y de que así no sería tan fácil, una vez que esto se hubiese logrado, producir el contragolpe y desplazar como muchas veces había sucedido a quienes se habían dado a la tarea de construir un camino distinto, un camino de emancipación y libertad. Se pensaba que era posible que cada país de nuestro subcontinente, con su propia historia, poniendo los píes y los sentidos sobre la tierra para responder a los rasgos propios, a su existencia como nación, para que entonces cada uno con su movimiento y capacidad, en la arqueo de su comunidad y la relación de sus sedimentos históricos, los países latinoamericanos en conjunto conseguirían una seria de cambios y transformaciones para sumarse al grupo de las naciones desarrolladas.

Entonces prevalecía por un lado la idea de homogeneidad y por otro la idea del cambio como suma del optimismo y la esperanza. Este esquema del pensamiento virtual y la realidad 
latinoamericana encontraban su germen en que no nos había ido tan mal económicamente. Contra lo que dicen los analistas ultraliberales, el mejor tiempo de crecimiento económico de América Latina se dio en los 25 años posteriores a la segunda guerra mundial. Con eso se propició la instalación de obras del estado de bienestar, que pudo culminar en un buen numero de nuestros países, proyectos que permitieron construir escuelas, universidades, impulsar el crecimiento de la producción industrial y conducir el empleo hacia niveles superiores, propiciar índices respetables de seguridad social, mejorar la salud y el bienestar de la población, realizar políticas de vivienda asequibles que permitieran a las clases medias en crecimiento tener acceso a un lugar digno para vivir, mientras al mismo tiempo las tasas demográficas se disparaban y los cinturones marginales que le daban un nuevo rostro de las poblaciones y ciudades en vías de convertirse en metrópolis, iban configurando con dureza el rostro del desengaño y del deterioro del modelo y del sistema.

Pero fueron también aquellos años un mejor tiempo para los países más prósperos. Se habla aún de los gloriosos 30, que vistos bajo la lupa de una posible explicación fueron años del soporte del desarrollo científico y tecnológico que definió el poder de los años posteriores, luego de que los aliados derrotaron al nazifacismo. "Y esos años fueron de enorme dinamismo, de expansión económica, de innovación tecnológica, de economías florecientes en sus números, y de crecimiento del PIB de los países más avanzados, que llegaron a crecer a ritmos del $5 \%$ promedio anual. En ese tiempo, nada virtual, los países Latinoamericanos crecieron en conjunto un poco más. Y llegaron a tasas de un poco más arriba del $5 \%$ regionalmente. En estas tres décadas hasta ya avanzado los setenta siguieron más o menos por esta ruta. Después las cosas no siguieron igual y eso hace el contraste de ésta mirada, de este acercamiento general sobre América Latina", que en ésta síntesis reconoce y busca acercarse a lo que paso con el territorio de la región continental.

Las ciudades, comenzando con Brasilia que se consolidaba como ejemplo de la modernidad a la que se aspiraba la sociedad latinoamericana conducida por las nuevas capas del poder económico, dejaba tras de si la huella de la contradicción. Al mismo que era construida esa urbe hoy ciudad patrimonio moderno de la humanidad, se fueron edificando las ciudades de los que la construyeron para no vivir en ella, por lo que fueron apareciendo, Taguatinga y livre_Bainderante. Otras como Sao Paulo y algunas ciudades más como Monterrey en México, recibían los alientos del desarrollo industrial, La Plata, su realización, hacia notar la pujanza de una Argentina de gran paso y peso en el continente, Lima buscaba recuperar su importancia regional entre las intermitencias de un Sendero Luminoso en camino, mientras otras más como Bogota y en general Colombia, El Salvador, Nicaragua, Guatemala, se veían envueltos en terribles circunstancias internas de lucha que costaron miles de vidas, pero que se pensaba allanarían paso a paso, aunque no sin las dificultades inherentes, el camino hacía la democracia.

Era observable en tal entorno, sin embargo, cómo esa América Latina, homogénea, esperanzada, con mucha más pobreza que ahora, porque sin duda éramos más pobres, se encontraba regada con la idea de que estábamos dando pasos que nos acercaban a una cierta emancipación nacional. Se respiraba en la vida nacional, dicho en términos amplios, la posibilidad de encontrar la ruta del progreso, no obstante ya por entonces en los más altos pisos del edificio del poder se comenzaba a revisar la idea del progreso infinito. Recordemos la respuesta del llamado Club de Roma, y posteriormente del Informe Brundtland, "Nuestro Futuro Común".

Retomamos lo que había pasado para dar salida a nuestro breve compendio inicial y observamos lo que ha pasado 45 años después. Y lo que cosntatamos es que en este espacio latinoamericano ubicado en el seno de la reestructuración de las relaciones internacionales de intercambio y cooperación, se suceden percepciones, sentimientos, subjetividades de unos países a otros, que dan sustento a concepciones distintas de aquellas que prevalecieron en la que fue la etapa que llamamos aquí de la esperanza. Y vemos así que hoy día América Latina 
al parecer no es ya una zona homogénea del mundo. Es más, en los principales seminarios y encuentros internacionales y el contexto y contenido de los trabajos más interesantes y originales que se han producido en los últimos tiempos, lo que se está preguntando es si sigue existiendo una América Latina, si sigue como una unidad así denominada, y no son pocos los que ponen en duda ésta reflexión. Es decir, se plantean dudas para explicar que ya no tenemos en conjunto las mismas razones para decir y mantener, para considerar o referir, que la región sigue siendo una entidad geográfica homogénea entre las regiones del mundo. Esto es algo que desde nuestro pinto de vista es valido pensar, aunque no estemos totalmente de acuerdo con su hipótesis de fondo.

Naturalmente no debemos olvidar que el idioma, los hechos compartidos en la historia, han marcado con ciertos rasgos culturales a nuestras naciones, y que en éstas recurren fenómenos en donde se verifican algunos paralelismos como es el caso del crecimiento demográfico y los problemas de nuestras ciudades, temas que tienen que ver con una racionalidad con la que se ha alimentado un modelo de desarrollo con el que también se vinculan los procesos y los problemas que le dan un carácter particular a este subcontinente.

Así, intentando contrastar las ideas en ésta intervención, algunos proponen diferentes formas de entender, de segmentar a América Latina para referirse a las tendencias que han seguido, y se muestran en su desarrollo o crecimiento. Vale retomar entonces el tema sobre nuestro ritmo de crecimiento, el que se mantuvo hasta cerca de los ochentas en aproximadamente un $5 \%$ promedio regional, para situar la evolución de las ideas. En los 18 años siguientes tuvimos el estallido de la crisis de la deuda por allí por el 82, que afectó hacia arriba y hacia abajo al continente en diferentes momentos, etapa en que se sufrieron condiciones de contracción productiva, de aumento de la pobreza, de déficit que se fueron acumulando, de involución en muchos países, y cuando se hizo una nueva medición hacia fines de los ochentas y principios de los noventas el crecimiento había

caído al $1.4 \%$ promedio anual.

Luego se sumaron años de resultados más pobres. Entre 1998 y el 2002 llegamos al $0.2 \%$, prácticamente nada y el per cápita se precipitó en caída libre. Los últimos tres años no han sido malos y ha habido una modesta etapa de recuperación especialmente por el impacto de la economía China en el ámbito internacional, la que, no obstante el debate sobre su esquema de "dumping" vía salarios y otras formas de subvención, su intervención en el ámbito internacional, en la economía global, trajo un cambio de los términos de intercambio de los productos latinoamericanos en el mercado. En los últimos tres años por esa vía y la de petróleo, hemos tenido un breve ciclo de recuperación en el que hemos llegado a alcanzar entre un $4 \%$ y un $4.5 \%$ de crecimiento anual, y sin embargo la realidad social es de pesimismo e incertidumbre.

El optimismo ya no forma parte de la estructura social presente. El resultado de un neoliberalismo más o menos eficaz al nivel de la macroeconomía al no pasar por las líneas de mejoramiento social de la población en su conjunto, no impide la incredulidad, dada la precariedad de su resultado en el reparto de la riqueza, lo que hace difícil pensar que pudiésemos vivir otro periodo de largo, o quizá mediano plazo, que nos permita transitar con otras medidas entre posibles opciones positivas de cambio, entre las condiciones impuestas por la globalización y sus agentes, que nos pudiesen llevar hacia un mejor itinerario cualitativo de desarrollo oportuno.

Hay un escepticismo en la América Latina de hoy que además se observa más segmentada. Todo esto ha despertado un nuevo fascinante debate sobre lo que hoy es la región, sobre que tan integrada se encuentra hoy América Latina. $Y$ en éste tema, siguiendo, y tomo las ideas de Luis Maira, se encuentran dos corrientes con dos versiones distintas con las que tratan de responder a éstas interrogantes. 
La primera es la tesis de las dos Américas Latinas, una América Latina del norte y otra del sur, separadas por el Canal de Panamá. La segunda es la de varias Américas Latinas en donde los elementos de mayor homogeneidad son los de cercanía y otras coincidencias. Una es la que se deriva de la que se da sólo entre los dos países más influyentes en la región. México y Brasil, la otra la que se da entre las subregiones que serían: la del caribe, la andina, la centroamericana, aunque en ésta última influyen México y Estados Unidos y la del cono sur. América Latina sería de ésta manera la relación y vínculos de diversos actores, su interacción entre la dinámica y las lógicas de sus posibles intereses, no obstante en ocasiones se encuentren como actores internacionales envueltos en lógicas diferentes. Veamos entonces estas dos versiones.

La idea de las dos Américas, la del norte y la del sur ha sido una idea sostenida por los académicos, intelectuales, diplomáticos y políticos brasileros. Alrededor de ésta hipótesis se estructura la idea de que hay dos cuerpos territoriales importantes que actúan e influyen en el esquema regional. La propuesta se basa en que las agendas de los países que están al norte, sus vínculos e intereses compartidos, su grado de dependencia y cercanía con los intereses de los Estados Unidos son diferentes a las de los países que se ubican en el sur, ya que éstos países del norte hacen parte importante del entorno geopolítico norteamericano y que la suscripción del Tratado de Libre Comercio, firmado por Estados Unidos y Canadá en 1993, llevó a México, estratégicamente, suscribir y encaminar al mismo tiempo lazos e intereses duraderos con éstos países. La extensa frontera entre éstos, a lo que se suma que se tiene en el caso mexicano un $70 \%$ o hasta un $80 \%$ del comercio de importación y exportación, concretan un relación fuertemente encadenada con la nación más poderosa del mundo.

Se entiende por esta razón que eso hace difícil entender un ritmo activo de intercambio e intereses con los vecinos del sur. En parte quizás los brasileros han impulsado la idea de que México sigue siendo un país latinoamericano, dada la importancia económica y el peso de la tradición y la cultura del país, quizás complementando en su favor, que tal cosa implica un apoyo para sí mismo y su liderazgo en la región por razones similares, supliendo la posible incoherencia de separar la realidad cultural e histórica de México de su situación económica y de las condiciones que se le vienen imponiendo.

Otro argumento entre los que sostienen ésta idea, es la de que hay fenómenos que son propios de los países al norte del Canal de Panamá que no tienen significación en los otros países del sur. Uno primordial es el de la centralidad del problema migratorio - que estamos seguros no se detendrá a pesar de lo ignominioso muro que ha ordenado construir la administración Bush y sus halcones - el sueño americano, no obstante la influencia de los medios masivos de comunicación en la adecuación de comportamientos, es más o menos irrelevante en la mayoría de los países del sur. En éstos se mira más hacia Europa.

Y es que cuando un país que como México recibe 18 mil millones de dólares al año, recursos que envían muchos de aquellos que viven irregularmente o legalmente, integrados todos en la economía estadounidense, las percepciones de la realidad se fundamenta y actúan de una forma concreta como cadenas de transmisión. O como en El Salvador, un pequeño país que recibe 1400 millones, y que entre los residuos de la guerra civil que le aterrorizo por décadas y los despojos de las dictaduras que le llenaron de calamidades, que lastimó tanto el futuro de muchos jóvenes, que de entre eso y otras cosas surgió la conocida "mara salvatrucha". Uno debe comprender que esas remesas, al igual que pasa con los otros países de la región pobre de Centroamérica, sirven para amortiguar los conflictos y posibles explosiones sociales, y son medios con los que se disfraza ó disimula lo que sería un conflicto desnudo. Esto, visto en su realidad, trae un tipo de relación de intercambio de conflicto de Estados Unidos, que no tienen los demás y que por lo tanto no están impactados tan directamente con algo que forma parte sustancial de éste problema que son los dólares que envían los migrantes a sus países, a sus familias, y que forman parte fundamental en la regulación de los problemas sociales. 
Es necesario precisar en este ámbito de ideas y realidades, que la situación de los países del área sur se visto con tal deterioro en la última década, que, como en el caso de Ecuador, Argentina y Perú, la migración hacia Europa, especialmente hacía España, ha aumentado y las remesas han adquirido en los últimos años vital importancia.

Pero en el caso de los territorios nacionales del sur se plantea la idea de que los problemas esquematizan factores distintos, a pesar de que las discontinuidades en el desarrollo y las complejidades en la cohesión territorial son similares, y es por eso que han logrado que entre 10 países del sur, con la adhesión de los que están a la cabeza del Caribe, como son Surinam y Guyana, acuerden una asociación sudamericana que comienza ya a tener una agenda común, con visos orgánicos, que siguen bajo cierta individualidad y al mismo tiempo convivencia, mostrando activa una especie de subregión con un proceso propio, con lo que se busca un protagonismo en el complejo mundo de la posguerra fría de las primeras décadas del siglo XXI.

Éstos países de América del sur, constituidos como asociación, trabajan sobre cuatro asuntos principales en los que convergen y pueden compartir problemas y soluciones con los demás: Primero, la necesidad de su integración física que significa, construir y compartir caminos, puertos y aeropuertos internacionales, comunes, con los cuales establecer la conectividad que sirva de enlace entre estos países, los que en gran medida han estado viviendo de espaldas, ya sea debido a realidades políticas, económicas o territoriales, y que recién hoy se convencen que en el mundo de la globalización que estamos viviendo, en el que se marca la redefinición tenaz y a veces dramática de los espacios territoriales, políticos y económicos (recordemos Irak, Afganistán o Los Balcanes), en donde sólo las grandes entidades regionales tienen posibilidad de protagonismo y participación en el mundo del futuro presente, obligan y hacen entonces necesario establecer y organizarse en cuerpos regionales en un mundo dominado por la estructuración de grandes regiones económicas y políticas, obviando y llevando consigo al mismo tiempo las particularidades culturales.

El gran tema es el tema energético. Cada uno de los países de América, en diferentes escalas o niveles tienen problemas de abastecimiento energético, por eso surge la idea de la integración energética. Hay en el sur dos grandes productores de gas y petróleo, Bolivia y Venezuela a lo que se suman las proporciones de Brasil, Ecuador y Argentina.

Por esa vía transcurre el proyecto de integrar la capacidad de recursos y resolver en gran medida el problema del abastecimiento y los precios para dinamizar las estrategias de desarrollo. De esto forma parte esencial la construcción del anillo energético que busca llevar el gas y el petróleo desde Venezuela hasta la Argentina, pasando por Brasil, contando con Bolivia en el otro extremo. Una obra monumental de más de 9 mil kilómetros de longitud, con un presupuesto inicial de 18 a 20 mil millones de dólares.

El tercer punto, demanda vital, es el de compartir los problemas de la agenda social, principalmente el de la pobreza, tanto como los de la superación de la desigualdad, que son el gran cáncer de América Latina. Interactuar por lo tanto en éstos problemas es fundamental para toda la región.

Un cuarto punto esencial a destacar, por igual para todos los países del norte y del sur, es el de la identidad cultural, el de las raíces históricas. Bien sabemos que en el mundo de la globalización quienes no sean capaces de defender su visión del hombre y de la historia, de la humanidad y la naturaleza, su acervo de creatividad, sus valores que dan sustancia a su identidad van a pasar a ser países, comunidades, sociedades masivas y subalternas en el renovado juego, como en el espectáculo visual y de contenidos del funcionamiento mundial. 
Así, quienes tienen y manifiestan, coinciden con estas visiones, consideran que estas dos Américas Latinas se irán poco a poco separando irremediablemente porque se sustentan en percepciones y lógicas distintas, pero que podrán mantener una inequívoca relación entre si, ya que se mueven en su interior esencias culturales, vínculos históricos y un idioma común.

Otra es la visión, la perspectiva, de quienes piensan que América Latina ya no existe homogéneamente, que su verdadera realidad no es la de dos grandes espacios geográficos, y que es no la de uno o dos sino que es la de varios actores. Quienes proponen esta segmentación parten de la noción de seis unidades regionales principales.

México y Brasil, los que por su peso económico y tamaño, extensión y riqueza en su biodiversidad, sus valores ecológicos, la fuerza de su cultura, constituyen y representan, por si mismos cada uno, una subregión; los países andinos, los centro americanos, los del caribe y los el cono sur. Se sostiene que sólo así se podría encontrar la tradicional concepción y los elementos que nos lleven a encontrar la vieja homogeneidad latinoamericana de los cincuentas y sesentas. Por esta ruta proponen la realización de esfuerzos comunes para tomar esa integración. Por esa vía se intento algún tiempo la ALALC.

Entendemos entonces que de todo esto surge la pregunta a cerca de cual es la mejor de éstas visiones. Contestar a esto es más difícil que establecer las divisiones, porque en el fondo cada una tiene su soporte y pueden ser complementarias si se camina en la dirección de objetivos comunes y proyectos unificadores. Asumir y darnos cuenta que es necesario aceptar que todo esto costará mucho trabajo y, sobre todo más apertura al diálogo para pensar y encontrar esa América Latina a la que seguimos afectiva e intelectualmente ligados, pero a cuya noción y realidad hay que darle una base material y acción conjunta en los escenarios internacionales y una gran dosis de cooperación económica y social que le constituyan en una región y sus naciones consultantes; y probablemente lo tendremos que hacer tomando en cuenta nuestras diferencias y, esperamos, fundamentalmente nuestras cualidades. En el reconocimiento de estos temas la relación de España, marco subrayando las palabras, es y deberá ser de compromiso, a pesar de que hoy, es posible que la necesidad así lo requiera, España ve más hacia la propia Europa y el este, que hacía América. Sin embargo no dejare de remarcar esto como lo he venido haciendo desde hace años.

Permítase entonces después de esta larga disección centrar este análisis de los problemas y los rasgos, los que a pesar de las particularidades siguen siendo parte de las grandes tendencias y por lo tanto de lo que deben ser las estrategias para superar los retos que enfrenta América Latina en los inicios del sigo XXI. Observar con cierta minuciosidad la encrucijada en la que se encuentra y que habrá de enfrentar inevitablemente. Quiero referirme entonces a cinco principales situaciones, que se significan grandes retos para el conjunto de nuestros países:

1. El reto del desarrollo social entre la pobreza y la desigualdad.

2. El escepticismo democrático y la necesidad de reformular los sistemas y las actuaciones de los grupos en la región, de los gobiernos y del sector privado y, también de los partidos políticos.

3. El debilitamiento de los actores sociales tradicionales y la necesidad de articular de mejor manera los nuevos actores sociales entre las contingencias del desarrollo.

4. El problema de la orientación que debe tener el desarrollo y aplicación de la ciencia, la tecnología y la educación superior.

5. Los dilemas de la integración propia e internacional de América Latina. 
En ésta división nada fortuita podemos iniciar con algunos datos duros que le imprimen una cierta claridad al tema. En el primer punto es imprescindible señalar la inequidad social y las lacras de la pobreza que son hoy día el acento más álgido y urgente por atender en la agenda de los países latinoamericanos. De su atención, del establecimiento de estrategias y acciones que resuelvan esto dependerán en gran parte todos los demás logros, sean políticos, económicos o culturales, que den veracidad y hagan realidad los cambios y soluciones pertinentes.

Reconocemos de partida que América Latina ha tenido en los últimos 25 años un inquietante aumento de su heterogeneidad social y productiva, cuyos resultados finales se han traducido, contradictoriamente, en un demoledor aumento de la pobreza y la desigualdad. Los datos son terriblemente impactantes. En víspera de la crisis de la deuda, allá por 1982, según datos de la CEPAL, había en la región 130 millones de pobres. Tuvimos las dificultades del crecimiento de la deuda con la que se fueron sepultando muchas de nuestras mejores expectativas. Luego pasamos por la llamada "década perdida", y al llegar al principio de los noventas, esos 130 millones de personas pobres se habían convertido en 190 millones.

Se produjo por entonces el gran cambio internacional, el hundimiento de la Unión Soviética, la terminación del campo socialista y la caída del muro de Berlín. Se generaron entonces muchas expectativas en el mundo - como hoy se hablan de los retos del milenio - se pensó de manera facilista respecto de la nueva sociedad y la igualdad en el mundo, igual se hizo en América Latina, lo hicieron tenazmente las iniciativas privadas y los sectores políticos de derecha. Pero nada de lo que se pensó como positivo sucedió así, porque cuando se hizo un nuevo corte en el 2003 los pobres que vivían en Latinoamérica eran ya 221 millones, y lo que era más dramático era que no se trataba de la pobreza común en el sentido conocido, sino que un gran numero de estos pobres se encontraban en la peor categoría de la pobreza, la de los indigentes, los de la extrema pobreza, que son aquellos a los que les alcanza para comer magramente una o quizás dos veces al día, ya no digamos para cubrir con dignidad su cuerpo y tener acceso a la salud, a la educación, a la vivienda, y a los beneficios del denominado desarrollo, el de la flexibilidad de la liberalización y la democracia.

Todo lo anterior mueve irremediablemente un panorama desalentador, al que uno puede sumar los casi 250 mil niños que trabajan en condiciones miserables y de desamparo, y lo cientos de miles de jóvenes urbanos que no alcanzan a ingresar a la universidad pública porque los presupuestos se han reducido año a año, y así también los miles de trabajadores que no encuentran empleo, o lo tienen con precariedad e inseguridad a través del las llamadas "outsourcing", temible situación especialmente cuando entramos en el proceso provocador del siglo $\mathrm{XXI}$.

Pero luego, entre el desaliento llegamos al 2003 y a pesar de que en los últimos tres años se ha mitigado un poco éste problema, podemos ver como una región que tenía tantas expectativas esperanzadoras en 1960, se metió en un túnel oscuro que lo llevó a pasar de 130 a 221 millones de pobres en menos de 20 años. Sin embargo en el fondo de estos datos duros sobre la pobreza lo más critico, la lacra más lacerante, es lo que corresponde a la desigualdad, porque América Latina, no se puede ocultar, no es la región más pobre del planeta. Hay países más pobres como lo del área sub-sahariana, pero nuestros índices de desarrollo humano son terriblemente contradictorios. Somos en suma la capital mundial de la desigualdad.

De brasil, por ejemplo, surgen datos inquietantes. Hay tiempos en que el $10 \%$ de la población más pobre solo obtiene el 1\% del ingreso nacional, mientras el $15 \%$ que representan los más ricos llegan al 52 o 54. En México no es diferente, allí la caída del salario en los últimos diez años a sido del $35 \%$ respecto de la llamada "canasta básica". 
Y uno encuentra, cuando se mete a profundidad en el problema, que esto es solo la punta del iceberg, que lo que sucede en Brasil, México, Colombia, Ecuador, Argentina, Bolivia - este último uno en los que la pobreza adquiere rango de indignidad y racismo, y aún así las quejas de las capas más conservadoras o aquellas ligadas a los sectores empresariales trasnacionales levantan la voz y traman en contra de la nacionalización a lo que tuenen derecho el pueblo boliviano - agregamos a los países centroamericanos y el cuadro se torna casi vergonzante.

Basta observar los índices de desarrollo humano y la desigualdad que tienen lugar en el territorio y las ciudades. En Argentina o en México uno se puede encontrar espacios de privilegio con habitantes cuyos ingresos per capita rebasan los 50 mil dólares, y cerca de allí entre distancias que no pasan los 20 kilómetros comunidades que sobreviven con menos de mil dólares. Zonas en el campo y la ciudad que combinan grupos minoritarios que han alcanzado ingresos parecidos a los que han llegado capas sociales medias y altas en los países de los denominados tigres asiáticos, que habitan entre contextos de modernidad, de prosperidad, que cuentan con espacios para el ocio y el consumo, para el turismo etc., y están allí contiguos a otros que viven ciclos de subsistencia.

La claridad de los datos, para quien los quiere ver con el espejo de la realidad y no a través del reflejo del grupo de Davos, presentan hoy día en el año 2006, a pesar de los aires tibios de los últimos 3 años en que la recuperación tuvo un respiro positivo, son datos escalofriantes y, para muchos, desalentadores. Es por eso que creemos que el futuro político de América Latina, no el de hoy o la próxima semana, estará marcado por los retos que plantea esta desigualdad, y por la necesidad objetiva de corregir esta situación, porque de no ser así esto nos llevara a un peligroso estado de condiciones en el que la realidad virtual y material de este panorama, puede llevar al sub-continente a situaciones nada deseables y arrastrar también a los que desde la poltrona del desarrollo, desde el desconocimiento o soslayo de la historia del progreso y la riqueza, sienten a salvo sin más y para siempre los privilegios.

El último informe de la FAO con motivo del Día mundial de la Alimentación, lo cual no deja de ser una sombría caída de la realidad, octubre 2006, deja al descubierto datos lamentables. En el mundo 850 millones personas sufren de hambre crónica, 52 millones en América Latina. En México el $30 \%$ de la población infantil padece desnutrición, mientras el país se encuentra entre los primeros lugares en rango de población que padece de obesidad. Ensambles socialmente contradictorios perviven y se encuentran en el territorio y las ciudades del mundo. Naturalmente, un primer paso es aceptar que las cosas no están como nos dicen los agentes que propagan la flexibilizar las normas y abundar en la libertad de los mercados, del bienestar excluyente, y sin embrago somos optimistas. Estamos convencidos que en esta medida habrá que capturar las oportunidades.

Una situación que es necesario retener y encontrar entre las opciones posibles, que no es nuevo, que es un dato histórico, es la concentración territorial y de la productividad social y económica en los mayores países de la región. Un destacado académico economista y político francés que fuera primer ministro por los años setentas Raymond Barre, que escribió un texto conocido, de gran influencia, "Los enclaves productivos en América Latina", y otro con el titulo de "Desarrollo económico: análisis y política", destacó en sus trabajos que América Latina es un continente donde existen en gran contradicción enclaves productivos e instalaciones industriales dotados de tecnologías de punta, islotes del primer mundo, formas de vida relacionadas, etc., y a pocos kilómetros lugares en donde siguen instaladas calidades y formas de vida y entidades productivas como a principios del siglo XX. Ya por los años cincuentas y sesentas Aldo Solari y Anibal Pinto al igual que luego Fernando Cardoso nos explicaron este fenómeno. 
Pero lo adicional a esto, lo que ha pasado, y a lo que hay que poner mucha atención es que tal situación se ha agravado. Esto consiste en que la modernidad y el atraso se han ido domiciliando de un modo contrastante en los mayores países, como México y Brasil.

El economista brasilero Winston Frisch, a principios de los años ochentas acuño un concepto que se hizo muy popular en la región para describir esa realidad de pobreza-riqueza, para describirla hablaba de Belindia, quería describir con cierta ironía, que existían áreas en donde se podían encontrar concentradas en lugares específicos todas las cualidades de espacialidad urbana y arquitectónica, de servicios, de formas productivas y de vida, como las que se pueden encontrar en el primer mundo, como podían encontrarse en Bélgica, espacios y personas concentrados en afinidad entre ellos, y otra parte muy grande del territorio como en la India colmada de pobreza y abandono.

Paradojas de la vida después de veinte años tal expresión ha encontrado su sentido en la India de Belindia, porque ahí encontramos desarrollos significativos, inversiones de transnacionales y de grupos internos de poder que son sectores puente de desarrollo significativo en el campo de la informática, que han hecho de una porción de la India un destacado lugar, dinámico e innovador, con impacto y presencia internacional entre los sectores de punta, mientras el resto de la India sigue siendo pobre, muy pobre y atascada entre condiciones de atraso y permanencias culturales de gran valor pero inundadas de miseria ambiental y de desarrollo.

En este sentido, valga el salto, quiero abordar el tema de México desde una perspectiva que puede desatar el debate o hilvanar los acuerdos. En un estudio reciente de académicos mexicanos en el que participó el mismo Luis Maira, destacado intelectual y político chileno actualmente embajador en Argentina, emergió el planteamiento de los cinco Méxicos. El México de la frontera norte, en el que habitan 16 y 17 millones de personas, el de las ciudades gemelas: Tijuana-San Isidro, Mexicali-Calexico, Laredo- Nuevo Laredo, Ciudad Juárez-El Paso, Matamoros-Brownsville, y otras más, en donde han convivido comunidades compartiendo hábitos y proyectos parecidos, pero que después del atentado del 11 de septiembre al World Trade Center han visto trastornado su proceso - agrego aquí y aprovecho este foro para expresar la profunda indignación y rechazo que nos merece el gobierno de George Bush por la aprobación para construir el ignominioso muro en la frontera mexicana con Estados Unidos, un muro más largo que el de Berlín que tanta lucha suscitó en Europa y en el mundo, mientras ante este otro la comunidad internacional mantiene un vergonzante silencio -. El segundo México es el que va de los estados de Sonora y Baja California hasta Tamaulipas, cuyo enclave industrial ha destacar es la ciudad de Monterrey, el norte del país que mantiene cercanía y comunicación e influencia cultural recíproca con el sur de los Estados Unidos.

El México central que va de Jalisco a Veracruz, en el eje de los puertos de Manzanillo, el puerto de Lázaro Cárdenas, donde se encuentra una de las acereras más importantes, hasta Veracruz en el Golfo de México, eje que atraviesa la Ciudad de México, y que es el sector más pululado y comunicado, dinámico, de la vida nacional, en el que se alternan riqueza y pobreza, miseria y atraso, así como una serie de ejemplos de los más destacado de la cultura ancestral, de la tradición, soporte y símbolo de identidad y nacionalismo cultural. El Pacífico conectado con los países del acuerdo del Pacífico.

Con un importante desarrollo turístico que se inicia en los cincuentas con Acapulco y sigue hasta el presente con lugares como Puerto Vallarta, Ixtapa y Puerto Escondido, que forman parte de éste circulo; mientras en el Golfo destacan, además de Veracruz, con su historia, los puertos petroleros y pesqueros de Ciudad Madero, Tampico, Alvarado y San Andrés Tuxtla; el cuarto México es el México pobre, el centroamericano que encuentra en la bahía de Tehuantepec su nicho y comprende los estados de Chiapas, Oaxaca y Tabasco y alcanza confines de Campeche y Yucatán, en donde los índices estudiados sobre morbilidad, nutrición, educación y en general todos los indicadores sociales, son los más lacerantes. 
El quinto México es el que se encuentra dentro de los Estados Unidos, los denominados hispanos, los latinoamericanos entre los cuales los de origen mexicano son inmensa mayoría, todos éstos suman ya el 14\%, 42 millones entre los 300 que son ya, y se prevé que para el 2050 sumaran el $25 \%$, habitantes que en el presente empiezan a moverse y a llevar a cabo expresiones populares de importancia reinvindicativa, como es el tema de Ley de migración, la misma que se encuentra entrampada en el Congreso de los Estados Unidos.

Para ejemplificar esto con algún punto de vista más, examinemos el problema migratorio ya que lo hemos tocado, porque cuando se presentan condiciones como el caso de México que recibe importantes remesas, como antes dijimos, el problema migratorio no es una cuestión de estadísticas o actitudes a favor o en contra, sino de factores que requieren de mejor atención. En Brasil por ejemplo la pobreza esta arriba en el noroeste y la prosperidad en la parte de abajo. La pobreza se encuentra en medio de un gigantesco rectángulo de millones de kilómetros cuadrados, que parte de Fortaleza, Manaos, se mete en el interior del Amazonas, sigue por Minas Gerais, Bello Horizonte y llega hasta Victoria. En este espacio viven millones de personas pobres, tan pobres o más como los de Nicaragua, Haití, Nigeria o Tanzania. Este es un fenómeno muy sobrecogedor. Y en este espacio el sueño no es ir a Estados Unidos, no es el sueño americano, aunque la globalización nos lo muestre así, sino es trasladarse a Río de Janeiro, Sao Paulo o Curitiba, quizá con suerte parar en Brasilia, ir a donde se encuentran las mayores oportunidades. Brasil un país enorme, podríamos decir un territorio continente de más de 8 millones de kilómetros cuadrados, que refleja esta concentración que hemos mencionado.

De esta forma, la existencia de nuestros países, de nuestras naciones de América Latina, de sus ciudades capitales, no lo podemos entenderlo como una simple convivencia, de concertación de acuerdos entre intereses de los que pueden representarse en estos.

Creemos con firmeza que la idea de una gran región en armonía y convivencia, sigue siendo el reto de América Latina, su formación e historia común, como lo ha sido no sin dificultades y obstáculos la formación de la Unión Europea. Y es entender esto en su cabal realidad y complejidad la primera condición, la exigencia, de una estrategia de desarrollo para superar los problemas actuales del conjunto, el paso necesario para arribar a mejores horizontes.

Para este examen, sintético como debe y puede ser una charla como ésta, permítaseme hacer una especie de corte geológico de las distintas realidades de América Latina, para plantearnos algunos problemas, en la línea de intentar la posible homogeneización e integración social que las estrategias compartidas de desarrollo posibles no pueden dejar de resolver. En este tan atrevido esquema la aplicación de realidades virtuales, de cara a posibles opciones de desarrollo desde donde se generen proyectos, nos refiere a puntos de valor crucial y decisivos, pues de otra manera una zona cuyo desarrollo demuestra una geometría social de inestabilidad, con gran diversidad cultural e identidad, tiene retos enormes para su inserción internacional y desarrollo local de gran equilibrio y cuyas respuestas a sus problemas, tomando en cuenta las condiciones particulares, requerirán un amplio espectro de proyectación de planes y programas que integren territorio, ciudades y procesos regionales, porque de no ser así América Latina continuara siendo una región con un conjunto de países, con ciudades y enclaves selectos, con riquezas abundantes, que van a seguir sumando una serie de crisis de resultados imprevisibles. Pero ésta mirada, como antes dije no es pesimista, las pautas de América Latina, un continente joven, de jóvenes, que ha superado grandes luchas, se encuentra también con oportunidades en los albores del siglo XXI, y para ello hay que superar las tendencias que acotan las fuerzas positivas, creadoras, intelectuales, que indudablemente existen. Para esto me voy a permitir expresar algunos puntos que desde nuestra perspectiva son vitales solucionar para recuperar la confianza y el optimismo, la pujanza del pasado. 
Ni duda cabe que América Latina vive tiempos de escepticismo democrático. Hace algunos años un grupo de científicos nuestros crearon una forma de medición que denominaron "latinobarómetro" y que mide entre otras cosas, la legitimidad de las instituciones de los países del continente, unos resultados más lamentables, en este rubro, fue en que en los diez países principales, fueron los partidos políticos, los parlamentos y los gobiernos los que sacaron la más baja calificación. Pero la constancia más importante de esto, es que, en algunos países que han logrado estructuras democráticas con cierta legitimidad, las encuestas demostraron que un porcentaje de la población se encontraba desencantada y que ponían como perspectiva la instauración de regímenes duros si se trataba de resolver problemas sociales. Las clases medias juegan en esto un papel fundamental, se encontraban diferencias entre país y país, naturalmente en el cono sur se ha avanzado, en la región andina se encuentran en curso otros procesos, mientras en Centro América y México, las derechas se han posesionado de controles esénciales aún utilizando estrategias inconfesables, como en el reciente caso de México.

Recordemos que los años setentas fueron años de gran nostalgia democrática, mientras permanecían dictaduras feroces y la represión tendía su manto de tortura (Centro América, cono sur, Brasil, Venezuela, el México del 68 y 71), la sociedad miraba con añoranza la falta de ciertas garantías entre las promesas del progreso. Las democracias liberales instauradas, superados aquellos años, conformaron el aliento de la esperanza y durante los ochentas, las democracias que devinieron en proyectos neoliberales se fueron llenando de estrategias macroeconómicas, como también de desequilibrios sociales. Algún autor llamó a este proceso como el de "democracia de baja intensidad".

Fue claro que los gobiernos elegidos de esa manera y los grupos privados que actuaban en el seno de su economía, utilizando los recursos públicos para beneficio de la privatización de los sectores estratégicos de la producción, fueron incapaces de producir los bienes públicos necesarios para modificar el estado de cosas. Resultado de esto, los movimientos populares han ido tomando lugar, los cuales convergen hoy en el movimiento alternativo antiglobalización - recordemos Puerto Alegre - y han provocado en las últimas décadas una cauda importante de revocatoria de mandatos presidenciales: Collor de Melo, Carlos Andrés Pérez, Fujimori, Mawuad, Bucaran, Alarcón, Sánchez de Lozada, La Rua y Menen, por mencionar algunos. (Rodrigo Borja) El reto en este rubro es, evidentemente, encontrar las fórmulas y acciones para reformar esta democracia, aún liberal, dotarla de los elementos necesarios, para que libre el abismo que le permita pasar de ser una democracia de propietarios hacia la construcción de ciudadanos. Alcanzar una estructura orgánica que se haga cargo no sólo de acceder al poder de ciertos grupos, sino de encontrar las fórmulas para resolver los ingentes problemas que le aprisionan y alcanzar una legitimidad más allá de las urnas.

Otro punto ha destacar es el debilitamiento de los sectores sociales de América Latina.

En España por ejemplo los actores sociales han conservado solidez y siguen actuantes. Hace 50 años, existían en nuestro subcontinente un vigoroso grupo de actores sociales, alimentado de individualidades. Sindicatos de trabajadores, agrupaciones sociales, confederaciones campesinas que lucharon por la Reforma Agraria y el reparto de la tierra, grupos estudiantiles organizados, que se hacían eco de muchas de las demandas sociales y de la proclamación del campo estructural. A principios del siglo XXI se puede notar como todo esto ha perdido vigor $\mathrm{y}$, habría que entender, no lo ha perdido solo por una anemia interna y por el decaimiento ideológico nacionalista, o por la ambigüedad en el compromiso social en el que ha caído una gran parte del sector intelectual. En esto es necesario insertar lo que significa el cambio del paso de la segunda a la tercera revolución industrial, los cambios en los procesos posindustriales a la automatización, la robotización, la superación del fordismo de los sectores de punta y la fragmentación de las partes del proceso e generación industrial, distribuida en países, que ha disminuido la capacidad negociadora de los trabajadores, de las agrupaciones sociales, ya de por si acotadas por la mediatización, la manipulación que se ejerce a través de los medios, la corrupción pública y privada y el progresivo abandono presupuestal para la 
educación pública y el desarrollo de la investigación científica y tecnológica. Para esto, bien sabemos, las agencias internacionales al servicio de la reconversión y reintegración del poder capitalista de los países y empresas centrales del poder han actuado con eficacia (Organización Mundial de Comercio, Banco Mundial, etc.), para controlar los procesos de innovación progresiva de la modernización o acceso a la posmodernidad de los países de la denominada periferia económica, a los que les imponen un carácter individualista en la educación, con el garlito de la competitividad, un cierto sabor elitista y privado para los comportamientos sociales y apoyo al debilitamiento de la gestión social.

Una última anotación en este sucinto esquema, es el tema de la defensa de la riqueza ecológica del continente. En ésta ruta la explotación de nuestros recursos por las agencias internacionales coludidos con grupos nacionales, locales, se entreveran inexorablemente con el crecimiento de ciudades en las que la contaminación ambiental, la especulación con el suelo urbanizable, el acelerado incremento demográfico, el uso inmoderado del territorio natural inmediato, el desempleo, etc., reciben el resultado como suma de la desigualdad y la pobreza.

Todo esto no es solo un problema de cifras, de millones de habitantes que conviven en los espacios urbanos, de índices de empleo o subempleo, de desempleo, de cambios en los procesos de salud-enfermedad de los habitantes, de problemas de género, de violencia e inseguridad, que se van acentuando, al igual que se cifras de la macroeconomía, de aumento de nuestras exportaciones - a pesar de que representen las de las maquiladoras - del aumento del precio de petróleo que beneficia a los países exportadores, o de localización y estudios cada vez minuciosos y detalladas a través del uso de los medios informáticos de los que hoy se disponen, de la focalización y desarrollo de proyectos puntuales, medibles con mayor exactitud para los factores de inversión, útiles para el financiamiento y recuperación, mejor conocimiento de la tasa de ganancia, o del posible bienestar social que pueden distribuir en una comunidad.

Lo virtual y el aprovechamiento de la red para recoger y compartir conocimiento (los datos sobre acceso a Internet y el espectro radioeléctrico mundial expresan también desigualdad en lo que algunos autores llaman info-pobres e info-ricos), comprende también el problema de lo que podríamos nombrar como la difusión y regreso de las epistemologías de la colonización. Este es un tema que bien podríamos debatir posteriormente. Porque el dato virtual expone y explica solo en aquello que tiene la capacidad posterior de llevar a cabo lo que supone como solución, lo que propugna como proceso aún encontrando las cuestiones de fondo. Pues más allá de la imagen y de la perspectiva que permite, la pervivencia de los problemas refiere compromisos esenciales en el que se identifiquen los valores, en el que se manifiestan el flujo de conocimiento vinculados a los fines colectivos.

Cuando se razona a cerca del tiempo en que vivimos, en ésta ocasión asumo y pido la disculpa por referirme al territorio Latinoamericano, cuando se cruzan en tales valoraciones positivo y negativo, cuando intentamos comprender que nos encontramos en el torbellino de cambios sustanciales hacia el futuro de la humanidad y en medio de una larga y dinámica transición en donde se gestiona la construcción de otros escenarios, recuerdo aquello que escribe José Manuel Naredo, tomando palabras de Max-Neef: “... mientras enfrentamos los muchos componentes de la megacrisis que se ha apoderado de nuestro mundo, padecemos de una especie de confusión generalizada cuando hacemos el esfuerzo de comprenderla.

Básicamente parecería que no logramos comprender en que consiste comprender, hemos alcanzado una etapa de nuestra historia que se caracteriza por el hecho de que sabemos mucho pero comprendemos muy poco... (Para añadir, a continuación, tres síntomas de nuestra confusión) nuestro compromiso con opciones de relevancia secundaria, la utilización de teorías simplistas para la interpretación de realidades sociales complejas, y el empobrecimiento o ambigüedad de nuestro lenguaje". 